\title{
Chattering-Free Adaptive Sliding Mode Control for Attitude Tracking of Spacecraft with External Disturbance
}

\author{
Xuxi Zhang, ${ }^{1,2}$ Xianping Liu, ${ }^{1}$ and Qidan $\mathrm{Zhu}^{2}$ \\ ${ }^{1}$ College of Science, Harbin Engineering University, Harbin 150001, China \\ ${ }^{2}$ College of Automation, Harbin Engineering University, Harbin 150001, China \\ Correspondence should be addressed to Xuxi Zhang; zxx08@126.com
}

Received 6 December 2013; Revised 9 February 2014; Accepted 13 February 2014; Published 17 March 2014

Academic Editor: Jun-Juh Yan

Copyright (c) 2014 Xuxi Zhang et al. This is an open access article distributed under the Creative Commons Attribution License, which permits unrestricted use, distribution, and reproduction in any medium, provided the original work is properly cited.

\begin{abstract}
The attitude tracking problem of spacecraft in the presence of unknown disturbance is investigated. By using the adaptive control technique and the Lyapunov stability theory, a chattering-free adaptive sliding mode control law is proposed for the attitude tracking problem of spacecraft with unknown disturbance. Simulation results are employed to demonstrate the effectiveness of the proposed control design technique in this paper.
\end{abstract}

\section{Introduction}

Attitude control of a spacecraft with unknown external disturbance has been studied intensively in the past decade. In [1], a general attitude control design framework was proposed for the analysis of attitude tracking control of a rigid body using the nonsingular unit quaternion representation. In [2], an adaptive control scheme for the attitude control of a rigid spacecraft was derived using a linear parameterization of the equation of motion, and global convergence of the tracking error to zero was shown using passivity theory. In [3], Tsiotras further proposed passivity based linear asymptotically stabilizing controllers without angular velocity feedback. In [4], Tayebi proposed a quaternion based dynamic output feedback control law of a rigid body without velocity measurement by introducing an auxiliary dynamical system. A typical feature in all of the mentioned attitude control schemes is that the control laws were all presented without considering external disturbances.

In order to deal with attitude control problem in the presence of unexpected disturbances, various approaches have been proposed in [5-7]. In [5], an inverse optimal adaptive control method was proposed to achieve attitude tracking and disturbance attenuation for a class of disturbances with bounded energy. In [6], an internal model method was presented for attitude tracking and disturbance rejection. In
[8-10], some sliding mode control strategies were proposed to solve the attitude control and disturbance rejection problem.

Sliding mode control method is known to be an efficient control technique applicable to systems with profound nonlinearity and modeling uncertainty [11]. The sliding mode control has several useful advantages such as fast response, low sensitivity to external disturbances, robustness to the plant uncertainties, and easy realization [12-14]. However, since the conventional sliding mode controllers often include the sign function, they suffer from the undesirable chattering phenomenon. A common method to alleviate chattering phenomenon is to insert a fixed or variable boundary layer near the sliding plane, such that a continuous control replaces the discontinuous one when the system is inside the boundary layer. Although the boundary layer method can give a chattering-free system, a finite steady-state error may exist. Then, other methods have increasingly gained researchers' interest, and several chattering-free control techniques have been studied in $[15,16]$. However, the chattering-free adaptive sliding mode control for attitude control of spacecraft in the presence of unknown disturbance has not been fully investigated, which constitutes the main motivation of the present research.

In this paper, we aim to propose a chattering-free adaptive sliding mode control law for the attitude tracking problem of rigid spacecraft in the presence of unknown disturbance. The 
main contribution of this paper is that (1) a dynamic sliding mode control law, which is free of chattering phenomenon, is presented for attitude tracking problem of rigid spacecraft and (2) the unknown external disturbance has been handled by using adaptive control technique.

The rest of this paper is organized as follows. The dynamic equations of spacecraft are briefly reviewed and the attitude tracking problem is formulated in Section 2. The chatteringfree adaptive sliding mode control law is given, and the stability analysis of closed-loop system is also presented in Section 3. A simulation example is provided to demonstrate the efficiency of the proposed control law in Section 4. Concluding remarks are collected in Section 5.

\section{Governing Equations and Problem Formulation}

In this section, we use the unit quaternion to represent the attitude of a rigid spacecraft. The advantage of quaternion representation for spacecraft attitude is due to its superior numerical property and void of singularity [17].

The dynamic model for the rigid spacecraft can be expressed as follows [6]:

$$
\begin{aligned}
{\left[\begin{array}{c}
\dot{q}_{v} \\
\dot{q}_{4}
\end{array}\right] } & =\frac{1}{2}\left[\begin{array}{c}
\left(q_{4} I_{3}+q_{v}^{\times}\right) \\
-q_{v}^{T}
\end{array}\right] \omega, \\
J \dot{\omega} & =-\omega^{\times} J \omega+u+d,
\end{aligned}
$$

where $\omega(t) \in \mathbb{R}^{3}$ denotes the angular velocity of the body fixed reference frame $\mathscr{F}$ with respect to an inertial reference frame $\mathscr{I}, u(t) \in \mathbb{R}^{3}$ and $d(t) \in \mathbb{R}^{3}$ are the control input and the external disturbance, respectively, $I_{3}$ is the $3 \times 3$ identity matrix, and $\mathbf{q}(t) \triangleq\left[q_{v}^{T}, q_{4}\right]^{T} \in \mathbb{R}^{3} \times \mathbb{R}$ represents the unit quaternion describing the orientation of the body fixed frame $\mathscr{F}$ with respect to the inertial frame $\mathscr{I}$, which is subject to the constraint

$$
q_{v}^{T} q_{v}+q_{4}^{2}=1
$$

The matrix

$$
J=\left[\begin{array}{lll}
J_{11} & J_{12} & J_{13} \\
J_{12} & J_{22} & J_{23} \\
J_{13} & J_{23} & J_{33}
\end{array}\right] \in \mathbb{R}^{3 \times 3}
$$

is the inertia matrix of the spacecraft, which is constant, symmetric positive definite, and the symbol $\times$ denotes an operator acting on any three-dimensional vector $v=\left[v_{1}, v_{2}, v_{3}\right]^{T}$ in such a way that

$$
v^{\times}=\left[\begin{array}{ccc}
0 & -v_{3} & v_{2} \\
v_{3} & 0 & -v_{1} \\
-v_{2} & v_{1} & 0
\end{array}\right] \in \mathbb{R}^{3 \times 3}
$$

In order to consider the attitude tracking problem, similar to [6], we assume that $\mathbf{q}_{d}(t) \triangleq\left[q_{d v}^{T}, q_{d 4}\right]^{T} \in \mathbb{R}^{3} \times \mathbb{R}$ with $q_{d v}^{T} q_{d v}+q_{d 4}^{2}=1$ and $\omega_{d}$ denotes the desired unit quaternion of the spacecraft in a desired body fixed reference frame
$\mathscr{F}_{d}$ with respect to the inertial frame $\mathscr{I}$ and the desired angular velocity of $\mathscr{F}_{d}$ with respect to $\mathscr{I}$ expressed in $\mathscr{F}_{d}$, respectively.

The dynamic equations corresponding to $\mathbf{q}_{d}(t)$ and $\omega_{d}$ are the following:

$$
\left[\begin{array}{c}
\dot{q}_{d v} \\
\dot{q}_{d 4}
\end{array}\right]=\frac{1}{2}\left[\begin{array}{c}
\left(q_{d 4} I_{3}+q_{d v}^{\times}\right) \\
-q_{d v}^{T}
\end{array}\right] \omega_{d}
$$

Next, as in [17], define the quaternion tracking error

$$
\mathbf{e}(t) \triangleq\left[e_{v}, e_{4}\right]^{T} \in \mathbb{R}^{3} \times \mathbb{R}
$$

with $e_{v}^{T} e_{v}+e_{4}^{2}=1$ as follows:

$$
\begin{aligned}
& e_{v}=q_{d 4} q_{v}-q_{4} q_{d v}-q_{d v}^{\times} q_{v}, \\
& e_{4}=q_{d v}^{T} q_{v}+q_{4} q_{d 4} .
\end{aligned}
$$

Then, the tracking error dynamics can be obtained as follows:

$$
\begin{aligned}
\dot{e}_{v} & =\frac{1}{2}\left(e_{4} I_{3}+e_{v}^{\times}\right) \bar{\omega}, \\
\dot{e}_{4} & =-\frac{1}{2} e_{v}^{T} \bar{\omega} \\
J \dot{\bar{\omega}} & =-\left(\bar{\omega}+R \omega_{d}\right)^{\times} J\left(\bar{\omega}+R \omega_{d}\right)+J\left(\bar{\omega}^{\times} R \omega_{d}-R \dot{\omega}_{d}\right)+u+d,
\end{aligned}
$$

where

$$
\bar{\omega}=\omega-R \omega_{d}
$$

is the error angular velocity of $\mathscr{F}$ relative to $\mathscr{F}_{d}$ expressed in $\mathscr{F}$ and $R\left(e_{v}, e_{4}\right)$ is the directional cosine matrix that brings $\mathscr{F}_{d}$ onto $\mathscr{F}$ as follows:

$$
R\left(e_{v}, e_{4}\right)=\left(e_{4}^{2}-e_{v}^{T} e_{v}\right) I_{3}+2 e_{v} e_{v}^{T}-2 e_{4} e_{v}^{\times} .
$$

Before proceeding further, we first introduce the following assumptions.

Assumption 1. The external disturbance $d(t)$ in (2) and its derivative $\dot{d}(t)$ are assumed to be bounded.

In this paper, we aim at attitude tracking in the presence of unknown disturbances. Given a desired quaternion $\mathbf{q}_{d}(t) \epsilon$ $Q_{u}=\left\{\left[q_{d v}^{T}, q_{d 4}\right]^{T} \in \mathbb{R}^{3} \times \mathbb{R} \mid q_{d v}^{T} q_{d v}+q_{d 4}^{2}=1\right\}$, the objective is to design a feedback controller such that, for any admissible disturbance satisfying Assumption 1 and for any $\mathbf{q}(0) \in Q_{u}$, the state of the closed-loop system composed of (9)-(11) and the controller is bounded and

$$
\lim _{t \rightarrow \infty} e_{v}=0, \quad \lim _{t \rightarrow \infty} \bar{\omega}=0 .
$$

\section{Main Result}

In order to convert the problem to a more tractable problem, we take the following coordinate transformation suggested in [5]:

$$
z=\bar{\omega}+K e_{v}
$$


where $K$ is a positive definite matrix which will be specified later.

Then, we obtain

$$
\begin{aligned}
\dot{z} & =\dot{\bar{\omega}}+K \dot{e}_{v} \\
& =f\left(\bar{\omega}, \omega_{d}, \dot{\omega}_{d}, \mathbf{e}\right)+\bar{u}+\bar{d}
\end{aligned}
$$

where

$$
\begin{aligned}
f\left(\bar{\omega}, \omega_{d}, \dot{\omega}_{d}, \mathbf{e}\right)= & -J^{-1}\left(\bar{\omega}+R \omega_{d}\right)^{\times} J\left(\bar{\omega}+R \omega_{d}\right) \\
& +\left(\bar{\omega}^{\times} R \omega_{d}-R \dot{\omega}_{d}\right)+\frac{1}{2} K\left(e_{4} I_{3}+e_{v}^{\times}\right) \bar{\omega} \\
\bar{u}= & J^{-1} u, \\
\bar{d}= & J^{-1} d .
\end{aligned}
$$

Remark 2. According to [6, Lemma 3.1], we need only to design a feedback controller such that the states of the closedloop system composed of (9), (10), (16), and the controller are bounded and $\lim _{t \rightarrow \infty} z(t)=0$.

In order to present an adaptive chattering-free sliding mode control law, we introduce the following dynamical sliding mode surfaces:

$$
\varrho(t)=\dot{S}(t)+\Lambda S(t),
$$

where $\Lambda$ is a positive definite matrix and $S(t)$ is defined as follows:

$$
S(t)=K_{1} \int_{0}^{t} z(\tau) d \tau+z(t),
$$

where $K_{1}$ is a positive definite matrix.

Remark 3. Based on Assumption 1, it is easy to see that

$$
\left\|\left(K_{1}+\Lambda\right) \bar{d}+\dot{\bar{d}}\right\| \leq C,
$$

where $C$ is an unknown positive constant.

Now, we are ready to state our main result.

Theorem 4. Under Assumption 1, let the adaptive sliding mode controller be

$$
\begin{aligned}
\dot{\bar{u}}= & -\left(K_{1}+\Lambda\right)\left(f\left(\bar{\omega}, \omega_{d}, \dot{\omega}_{d}, \mathbf{e}\right)+\bar{u}\right)-\dot{f}\left(\bar{\omega}, \omega_{d}, \dot{\omega}_{d}, \mathbf{e}\right) \\
& -\Lambda K_{1} z-\widehat{C} \operatorname{sgn}(\varrho)-K_{2} \varrho, \\
\dot{\widehat{C}}= & \sum_{i=1}^{3}\left|\varrho_{i}\right|,
\end{aligned}
$$

where $K_{2}$ is a positive definite matrix, $\widehat{C}$ is the estimation of the unknown constant $C$, and

$$
\operatorname{sgn}(\varrho)=\left[\operatorname{sgn}\left(\varrho_{1}\right), \operatorname{sgn}\left(\varrho_{2}\right), \operatorname{sgn}\left(\varrho_{3}\right)\right]^{T} .
$$

Then, for any initial condition, the states of the closed-loop system composed of (9), (10), (16), and (21) are bounded and

$$
\lim _{t \rightarrow \infty} z(t)=0 \text {. }
$$

Proof. Firstly, consider the following Lyapunov function candidate:

$$
V=\frac{1}{2}\left[\varrho^{T} \varrho+\widetilde{C}^{2}\right]
$$

where $\widetilde{C}=\widehat{C}-C$.

Taking time derivative of the Lyapunov function candidate $V$, one obtains that

$$
\dot{V}=\varrho^{T} \dot{\varrho}+(\widehat{C}-C) \dot{\widehat{C}}
$$

Combining (25) with (18) and (19), it follows that

$$
\begin{aligned}
\dot{V} & =\varrho^{T}(\ddot{S}(t)+\Lambda \dot{S}(t))+(\widehat{C}-C) \dot{\widehat{C}} \\
& =\varrho^{T}\left(K_{1} \dot{z}+\ddot{z}+\Lambda\left(K_{1} z+\dot{z}\right)\right)+(\widehat{C}-C) \dot{\widehat{C}} \\
& =\varrho^{T}\left(\Lambda K_{1} z+\left(K_{1}+\Lambda\right) \dot{z}+\ddot{z}\right)+(\widehat{C}-C) \dot{\widehat{C}} .
\end{aligned}
$$

Inserting (16) into the right hand of (26), it yields

$$
\begin{aligned}
\dot{V}= & \varrho^{T}\left(\Lambda K_{1} z+\left(K_{1}+\Lambda\right)(f+\bar{u}+\bar{d})+\dot{f}+\dot{\bar{u}}+\dot{\bar{d}}\right) \\
& +(\widehat{C}-C) \dot{\widehat{C}} .
\end{aligned}
$$

According to (20), we have

$$
\begin{aligned}
\dot{V}= & \varrho^{T}\left(\Lambda K_{1} z+\left(K_{1}+\Lambda\right)(f+\bar{u})+\dot{f}+\dot{\bar{u}}\right. \\
& \left.+\left(K_{1}+\Lambda\right) \bar{d}+\dot{\bar{d}}\right)+(\widehat{C}-C) \dot{\widehat{C}} \\
\leq & \varrho^{T}\left(\Lambda K_{1} z+\left(K_{1}+\Lambda\right)(f+\bar{u})+\dot{f}+\dot{\bar{u}}\right) \\
& +C\|\varrho\|+(\widehat{C}-C) \dot{\widehat{C}} .
\end{aligned}
$$

Furthermore, introducing the adaptive sliding mode controller (21) into the right hand of inequality (28), one can obtain

$$
\begin{aligned}
\dot{V} & \leq \varrho^{T}\left(-K_{2} \varrho-\widehat{C} \operatorname{sgn}(\varrho)\right)+C\|\varrho\|+(\widehat{C}-C) \dot{\widehat{C}} \\
& \leq-\varrho^{T} K_{2} \varrho-\widehat{C} \sum_{i=1}^{3}\left|\varrho_{i}\right|+C\|\varrho\|+(\widehat{C}-C) \dot{\widehat{C}} \\
& \leq-\varrho^{T} K_{2} \varrho+C\|\varrho\|-C \sum_{i=1}^{3}\left|\varrho_{i}\right| \\
& \leq-\lambda_{\max }\left(K_{2}\right)\|\varrho\|^{2},
\end{aligned}
$$

where $\lambda_{\max }\left(K_{2}\right)$ denotes the largest eigenvalue of the positive definite matrix $K_{2}$.

Therefore, the states $\varrho$ and $\widetilde{C}$ are bounded. By the construction of $\varrho$ and $S$, it is easy to conclude that $z$ is bounded. Furthermore, by the coordinate transformation (15), $\bar{\omega}$ is also bounded. Next, let $\alpha(t)=\lambda_{\text {max }}\left(K_{2}\right)\|\varrho(t)\|^{2}$. Integrating both sides of (29) yields that

$$
\int_{0}^{t} \alpha(\tau) d \tau \leq V(0)-V(t) \leq V(0) .
$$


Thus, $\lim _{t \rightarrow+\infty} \int_{0}^{t} \alpha(\tau) d \tau$ exists and is finite. On the other hand, $\dot{\alpha}(t)$ is bounded for all $t \geq 0$ since all states are bounded. Thus, by using Barbalat's lemma, one has

$$
\lim _{t \rightarrow+\infty} \alpha(t)=0
$$

and therefore

$$
\lim _{t \rightarrow+\infty} \varrho(t)=0 \text {, }
$$

which implies that

$$
\lim _{t \rightarrow+\infty} z(t)=0
$$

Remark 5. In the design of traditional sliding mode control, the sliding mode surface is often defined as

$$
\varrho(t)=z(t)
$$

and then under the assumption that $\|\bar{d}\| \leq C$, the adaptive sliding mode controller can be designed as

$$
\begin{gathered}
\bar{u}=f\left(\bar{\omega}, \omega_{d}, \dot{\omega}_{d}, \mathbf{e}\right)-K \varrho-\widehat{C} \operatorname{sgn}(\varrho), \\
\dot{\widehat{C}}=\sum_{i=1}^{3}\left|\varrho_{i}\right|,
\end{gathered}
$$

where $K$ is a positive definite matrix, $\widehat{C}$ is the estimation of the unknown constant $C$, and

$$
\operatorname{sgn}(\varrho)=\left[\operatorname{sgn}\left(\varrho_{1}\right), \operatorname{sgn}\left(\varrho_{2}\right), \operatorname{sgn}\left(\varrho_{3}\right)\right]^{T} .
$$

The difference between the adaptive sliding mode controllers (21) and (35) is that the discontinuous sign function appears in the first derivative of the controller (21); thus chattering phenomenon is alleviated.

\section{Numerical Simulation}

In this section, we present a numerical simulation example to illustrate the effectiveness of the developed adaptive chattering-free sliding mode control design method for attitude tracking of rigid spacecraft.

The simulation parameters are taken from [18] with some adjustments. The inertial matrix $J$ of the spacecraft and disturbance $d$ are assumed to be

$$
\begin{gathered}
J=\left[\begin{array}{ccc}
1 & 0 & 0 \\
0 & 0.63 & 0 \\
0 & 0 & 0.85
\end{array}\right], \\
d=\left[\begin{array}{c}
0.2 \sin (0.2 t) \\
0.1 \sin (0.1 t) \\
0.2 \sin (0.3 t)
\end{array}\right] \mathrm{Nm},
\end{gathered}
$$

respectively. In order to show the effectiveness of the proposed attitude tracking control law, we suppose that the

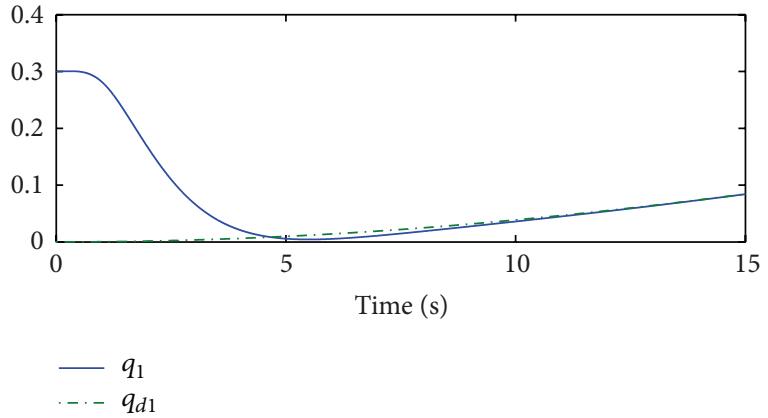

(a)

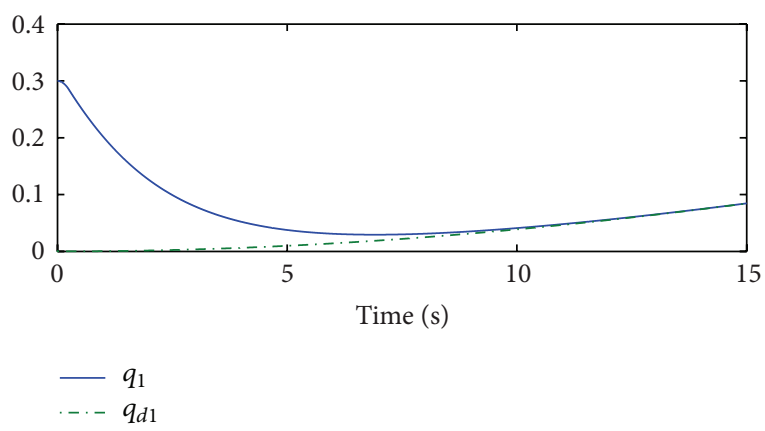

(b)

FIGURE 1: Comparison of attitude quaternion tracking: (a) controller (21) and (b) controller (35).

desired unit quaternion to be tracked is generated by dynamic equation (6), in which the desired angular velocity is given by

$$
\omega_{d}(t)=0.05\left[\sin \left(\frac{\pi}{100} t\right), \sin \left(\frac{2 \pi}{100} t\right), \sin \left(\frac{3 \pi}{100} t\right)\right]^{T} \mathrm{rad} / \mathrm{s}
$$

Note that the disturbance in the form of (38) is very standard [8], and it is easy to verify that Assumption 1 is satisfied; that is, there is an unknown positive constant $C$ such that $\left\|\left(K_{1}+\Lambda\right) \bar{d}+\dot{\bar{d}}\right\| \leq C$ for some selected control gain $K_{1}$ and $\Lambda$. Therefore, according to Theorem 4 , we can get the adaptive sliding mode control law as in (21). The simulation results are shown in Figures 1, 2, 3, 4, 5, 6, 7, and 8, where the initial value of unit quaternion $q(0)$ and the initial value of the angular velocity $\omega(0)$ are given by

$$
\begin{gathered}
q(0)=[0.3,-0.2,-0.3,0.8832]^{T}, \\
\omega(0)=[0,0,0]^{T} \mathrm{rad} / \mathrm{s},
\end{gathered}
$$

respectively. The initial desired unit quaternion $\mathbf{q}_{d}(0)$ is given by

$$
\mathbf{q}_{d}(0)=[0,0,0,1]^{T},
$$

and the initial values of the remaining states are all chosen as 0 .

The initial values and parameter matrices of the adaptive sliding mode control law (21) are chosen to be 


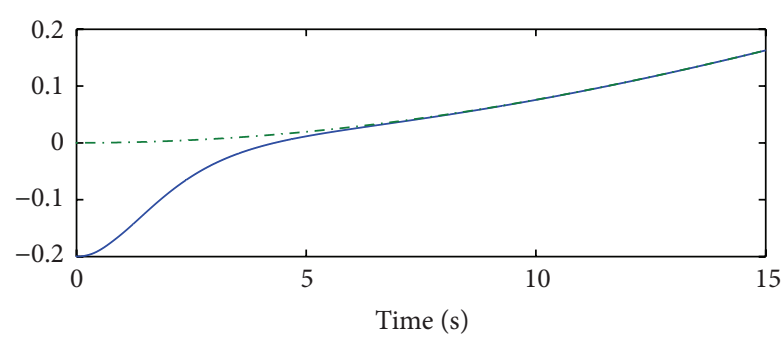

$$
\begin{aligned}
& -q_{2} \\
& --q_{d 2}
\end{aligned}
$$

(a)

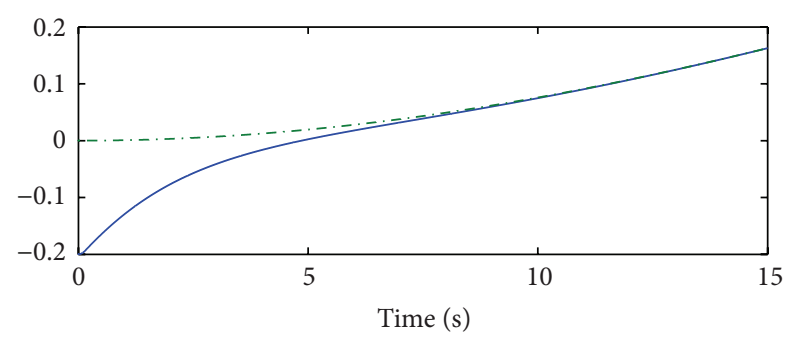

$$
q_{2}
$$$$
\text { -.. } q_{d 2}
$$

(b)

FIGURE 2: Comparison of attitude quaternion tracking: (a) controller (21) and (b) controller (35).

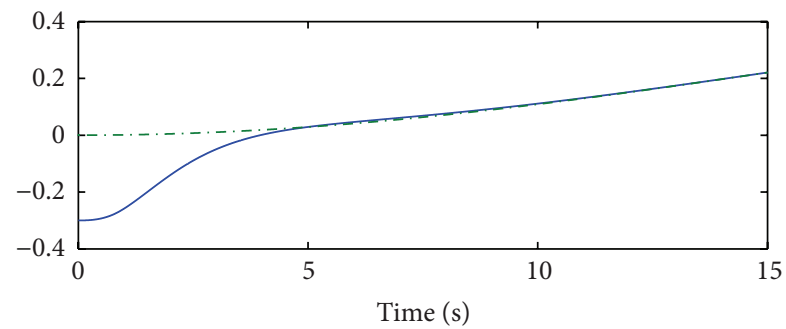

$-q_{3}$

$$
\cdot . \cdot-q_{d 3}
$$

(a)

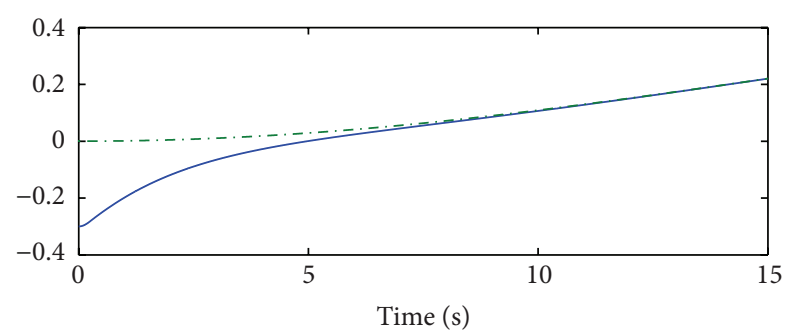

$$
-q_{3}
$$$$
\cdot-\cdot q_{d 3}
$$

(b)

FIgURE 3: Comparison of attitude quaternion tracking: (a) controller (21) and (b) controller (35).

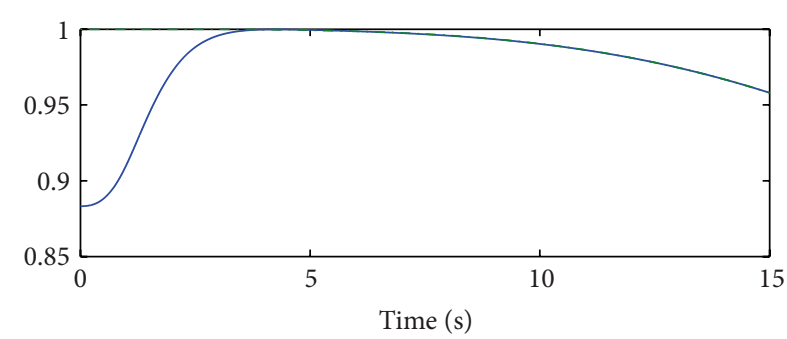

$$
\begin{array}{ll}
- & q_{4} \\
-- & q_{d 4}
\end{array}
$$

(a)

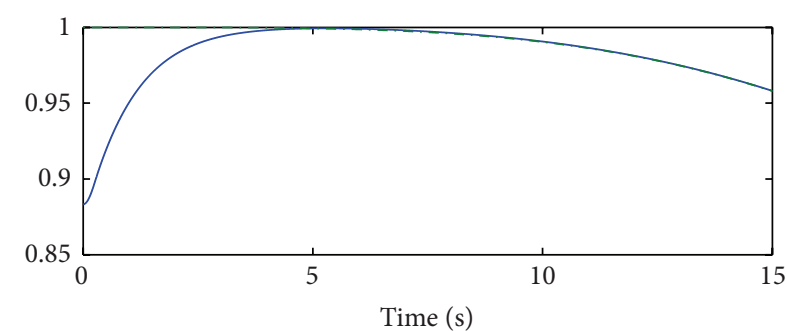

$-q_{4}$

(b)

FIGURE 4: Comparison of attitude quaternion tracking: (a) controller (21) and (b) controller (35).

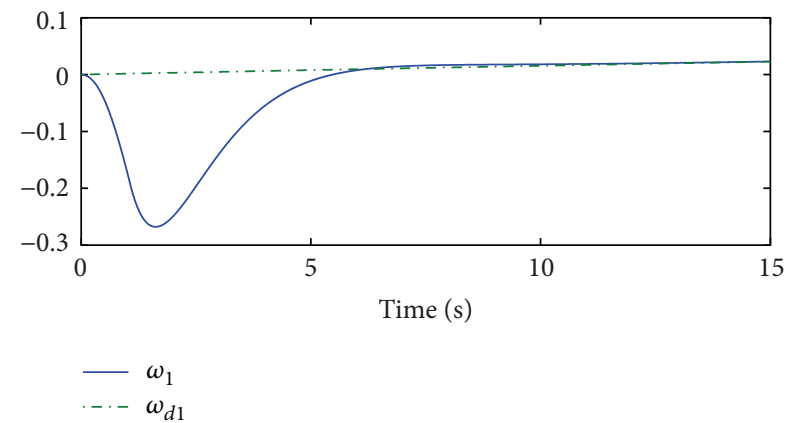

(a)

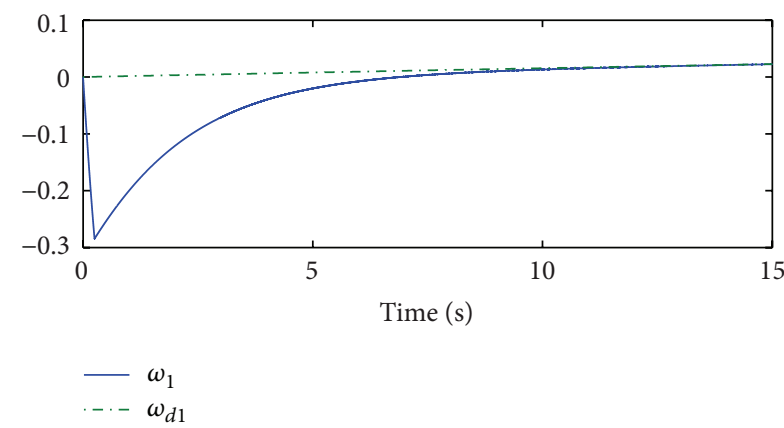

(b)

FIGURE 5: Comparison of angular velocity tracking: (a) controller (21) and (b) controller (35). 


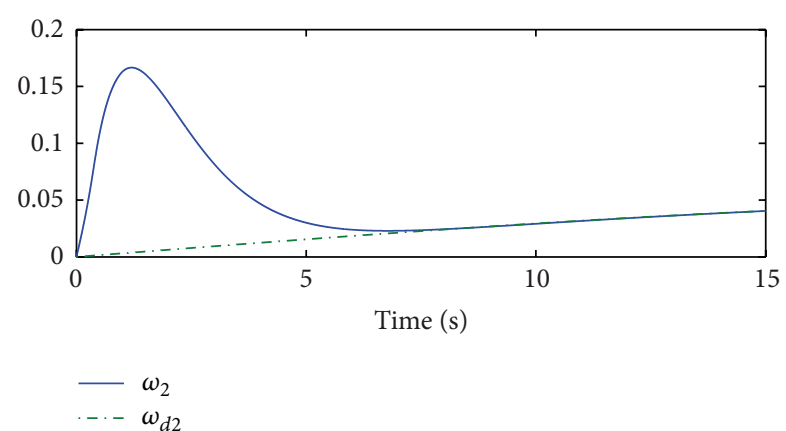

(a)

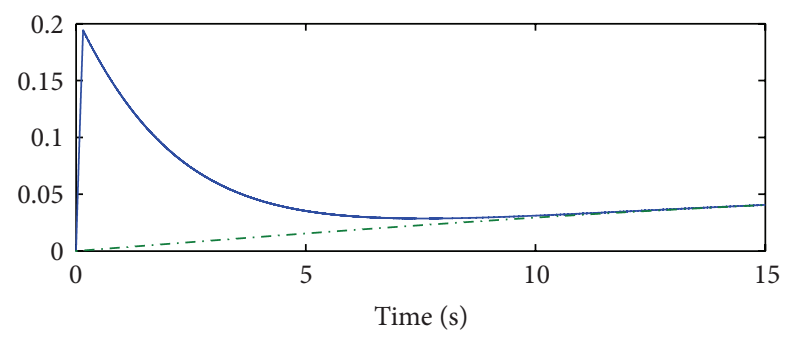

$$
-\omega_{2}
$$

(b)

Figure 6: Comparison of angular velocity tracking: (a) controller (21) and (b) controller (35).

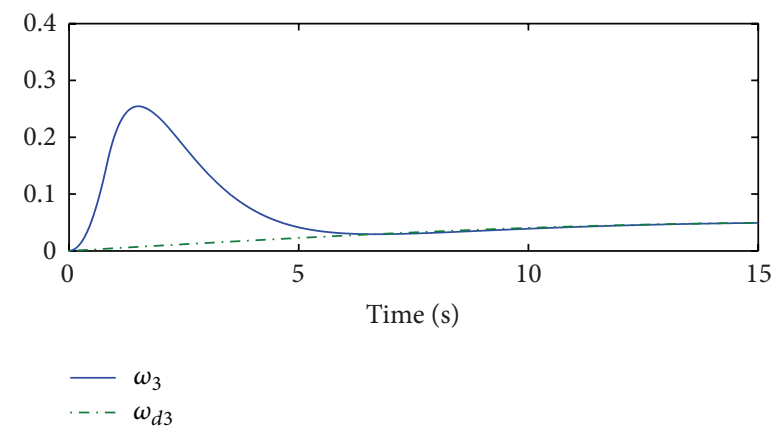

(a)

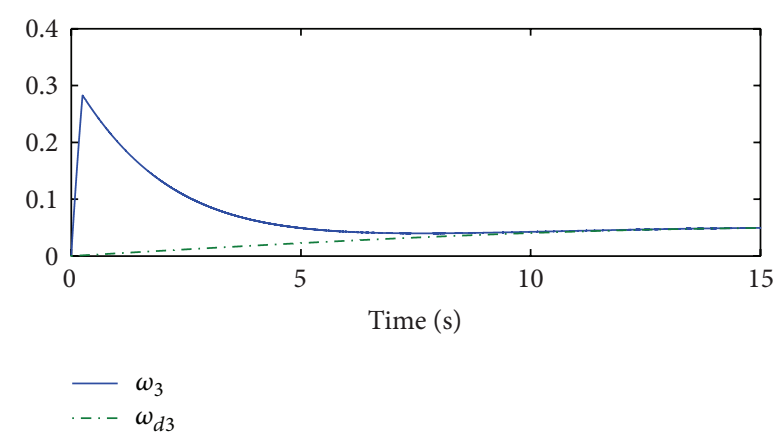

(b)

FIGURE 7: Comparison of angular velocity tracking: (a) controller (21) and (b) controller (35).

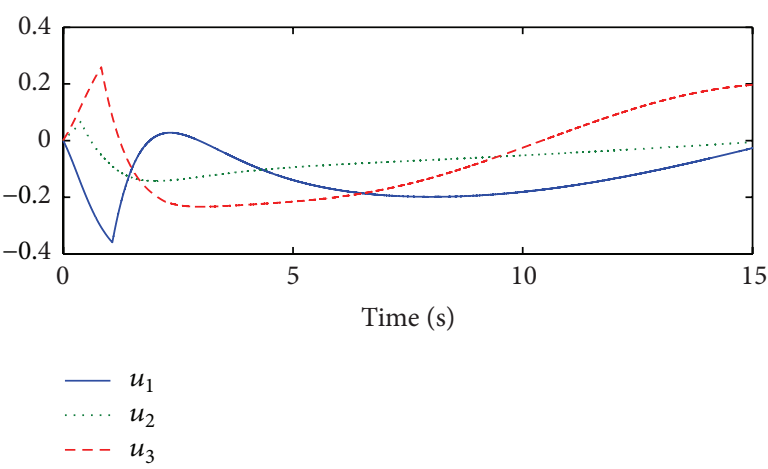

(a)

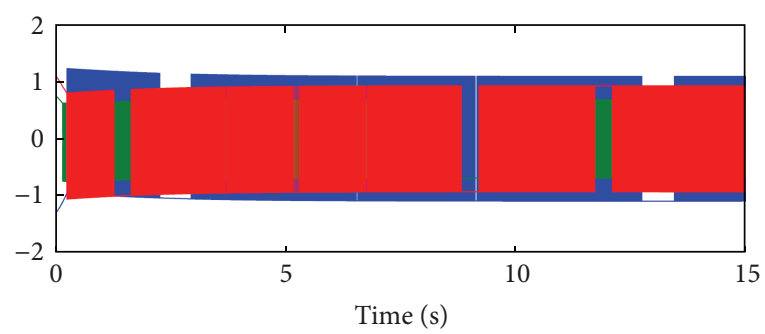

$\begin{array}{ll}- & u_{1} \\ \ldots \ldots & u_{2} \\ --- & u_{3}\end{array}$

(b)

Figure 8: Comparison of control input: (a) controller (21) and (b) controller (35).

$\bar{u}(0)=[0,0,0]^{T}, \widehat{C}(0)=1, K=2 I, K_{1}=I, K_{2}=I, \Lambda=I$. In order to show the effectiveness of the proposed controller, the comparison of simulation results between controllers (21) and (35) are presented in Figures 1-8 where the initial values and parameter matrices of the adaptive sliding mode control law (35) are chosen to be $\widehat{C}(0)=1$ and $K=I$. (a) of Figures $1-8$ is the results of the adaptive sliding mode controller (21), while (b) of Figures 1-8 is the results of the adaptive sliding mode controller (35). The attitude tracking of quaternion is shown in Figures 1-4, which show that the adaptive sliding mode control law (21) proposed in this paper guaranteed attitude tracking of spacecraft in the presence of the disturbance. Figures 5-7 depict the time response of angular velocity of the spacecraft. The time response of control torques is illustrated in Figure 8, which show that the adaptive sliding mode control law (21) is free of chattering.

\section{Conclusion}

The attitude tracking problem of spacecraft with unknown disturbance has been studied in this paper. The disturbance $d$ and its derivative $\dot{d}$ involved in the spacecraft model have been assumed to be bounded with unknown upper boundary. By combining the techniques from sliding mode control and robust adaptive control, a chattering-free adaptive sliding mode attitude tracking control law has been presented with the help of a dynamical sliding mode surface. 
Finally, a numerical example has been provided to show the usefulness and effectiveness of the proposed design method.

\section{Conflict of Interests}

The authors declare that there is no conflict of interests regarding the publication of this paper.

\section{Acknowledgments}

This work was supported by the China Postdoctoral Science Foundation funded project under Grant no. 2013M531016 and the Fundamental Research Funds for the Central Universities.

\section{References}

[1] J. T.-Y. Wen and K. Kreutz-Delgado, "The attitude control problem," IEEE Transactions on Automatic Control, vol. 36, no. 10, pp. 1148-1162, 1991.

[2] O. Egeland and J.-M. Godhavn, "Passivity-based adaptive attitude control of a rigid spacecraft," IEEE Transactions on Automatic Control, vol. 39, no. 4, pp. 842-846, 1994.

[3] P. Tsiotras, "Further passivity results for the attitude control problem," IEEE Transactions on Automatic Control, vol. 43, no. 11, pp. 1597-1600, 1998.

[4] A. Tayebi, "Unit quaternion-based output feedback for the attitude tracking problem," IEEE Transactions on Automatic Control, vol. 53, no. 6, pp. 1516-1520, 2008.

[5] W. Luo, Y.-C. Chu, and K.-V. Ling, "Inverse optimal adaptive control for attitude tracking of spacecraft," IEEE Transactions on Automatic Control, vol. 50, no. 11, pp. 1639-1654, 2005.

[6] Z. Chen and J. Huang, "Attitude tracking and disturbance rejection of rigid spacecraft by adaptive control," IEEE Transactions on Automatic Control, vol. 54, no. 3, pp. 600-605, 2009.

[7] X. Zhang and D. Cheng, "Nonlinear internal model based attitude tracking and disturbance rejection," Asian Journal of Control, vol. 14, no. 5, pp. 1397-1402, 2012.

[8] Z. Zhu, Y. Xia, and M. Fu, "Adaptive sliding mode control for attitude stabilization with actuator saturation," IEEE Transactions on Industrial Electronics, vol. 58, no. 10, pp. 4898-4907, 2011.

[9] A.-M. Zou, K. D. Kumar, Z.-G. Hou, and X. Liu, "Finite-time attitude tracking control for spacecraft using terminal sliding mode and chebyshev neural network," IEEE Transactions on Systems, Man, and Cybernetics B, vol. 41, no. 4, pp. 950-963, 2011.

[10] K. Lu, Y. Xia, Z. Zhu, and M. V. Basin, "Sliding mode attitude tracking of rigid spacecraft with disturbances," Journal of the Franklin Institute, vol. 349, no. 2, pp. 413-440, 2012.

[11] J. Y. Hung, W. Gao, and J. C. Hung, "Variable structure control. A survey," IEEE Transactions on Industrial Electronics, vol. 40, no. 1, pp. 2-22, 1993.

[12] M. Chen and W.-H. Chen, "Sliding mode control for a class of uncertain nonlinear system based on disturbance observer," International Journal of Adaptive Control and Signal Processing, vol. 24, no. 1, pp. 51-64, 2010.

[13] T. R. Oliveira, A. J. Peixoto, E. V. L. Nunes, and L. Hsu, "Control of uncertain nonlinear systems with arbitrary relative degree and unknown control direction using sliding modes,"
International Journal of Adaptive Control and Signal Processing, vol. 21, no. 8-9, pp. 692-707, 2007.

[14] A. J. Koshkouei, "Passivity-based sliding mode control for nonlinear systems," International Journal of Adaptive Control and Signal Processing, vol. 22, no. 9, pp. 859-874, 2008.

[15] M. Falahpoor, M. Ataei, and A. Kiyoumarsi, "A chattering-free sliding mode control design for uncertain chaotic systems," Chaos, Solitons and Fractals, vol. 42, no. 3, pp. 1755-1765, 2009.

[16] H. Li, X. Liao, C. Li, and C. Li, "Chaos control and synchronization via a novel chatter free sliding mode control strategy," Neurocomputing, vol. 74, no. 17, pp. 3212-3222, 2011.

[17] M. D. Shuster, "A survey of attitude representations," Journal of the Astronautical Sciences, vol. 41, no. 4, pp. 439-517, 1993.

[18] F. Lizarralde and J. T. Wen, "Attitude control without angular velocity measurement: a passivity approach," IEEE Transactions on Automatic Control, vol. 41, no. 3, pp. 468-472, 1996. 


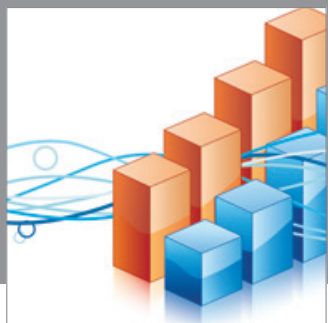

Advances in

Operations Research

mansans

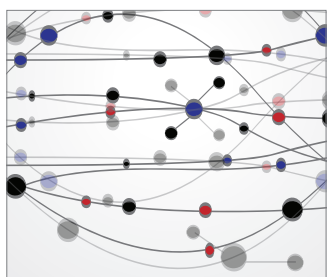

The Scientific World Journal
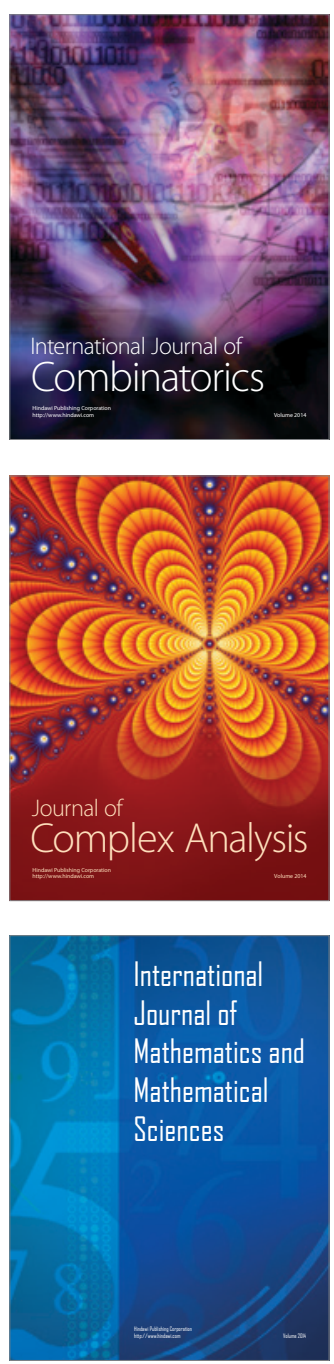
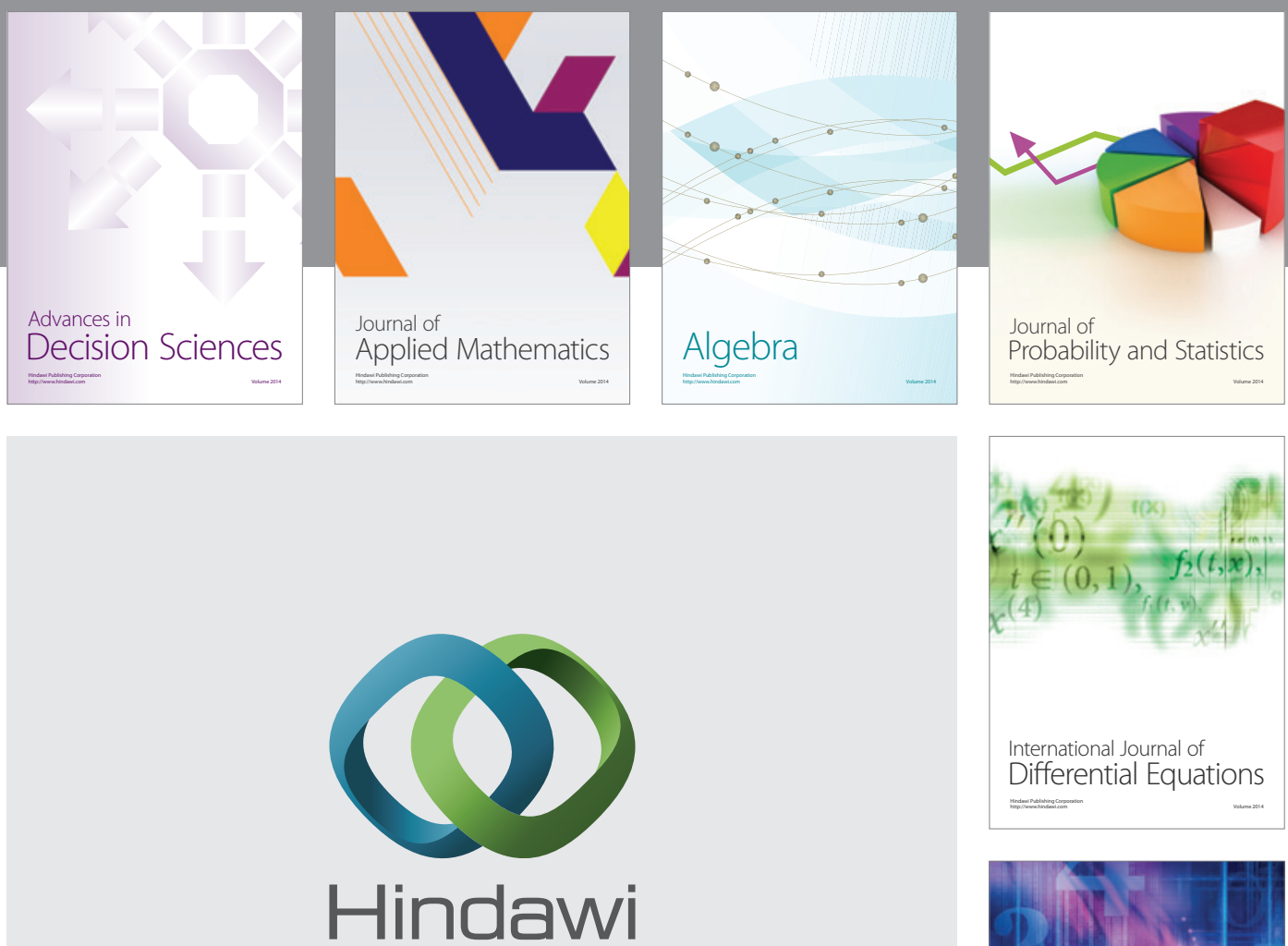

Submit your manuscripts at http://www.hindawi.com
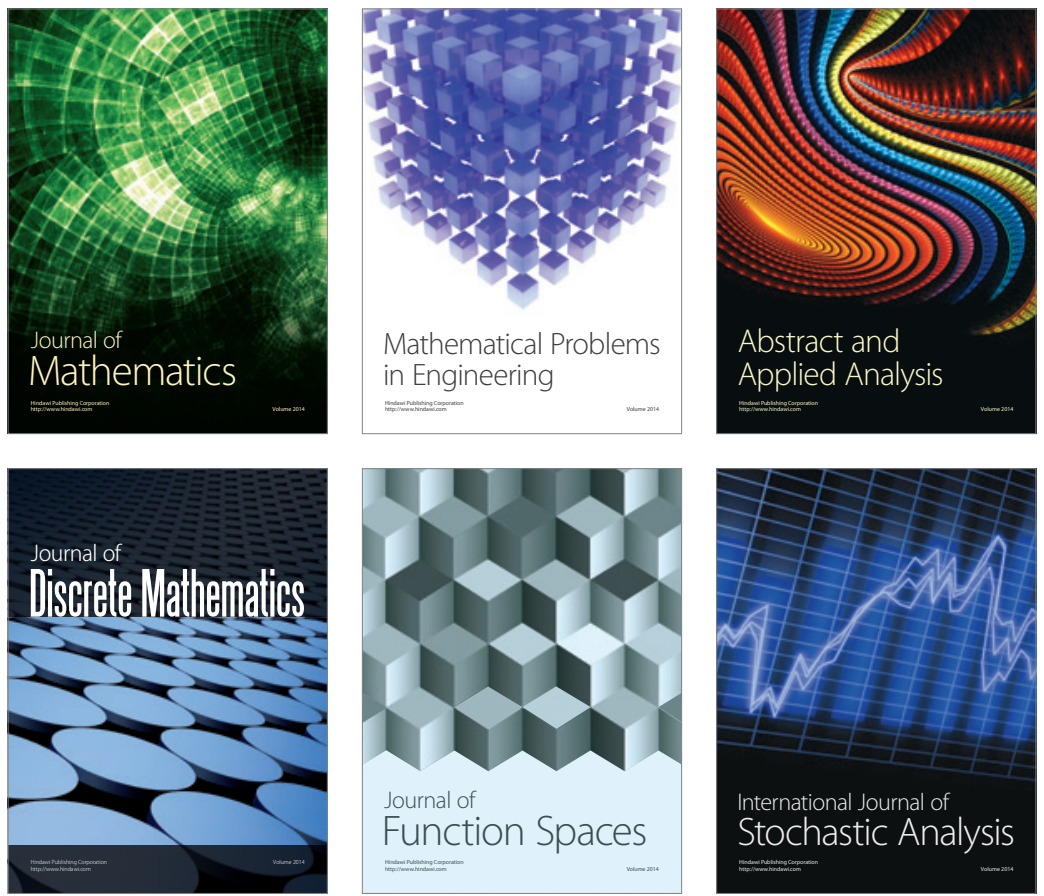

Journal of

Function Spaces

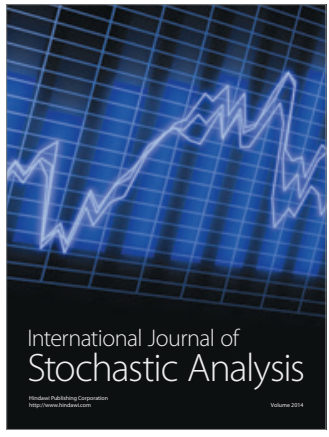

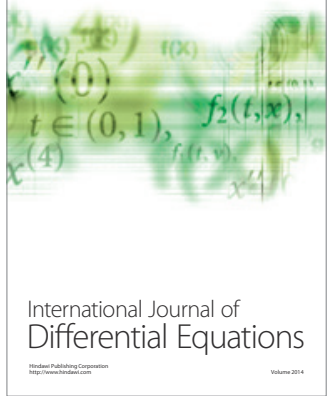
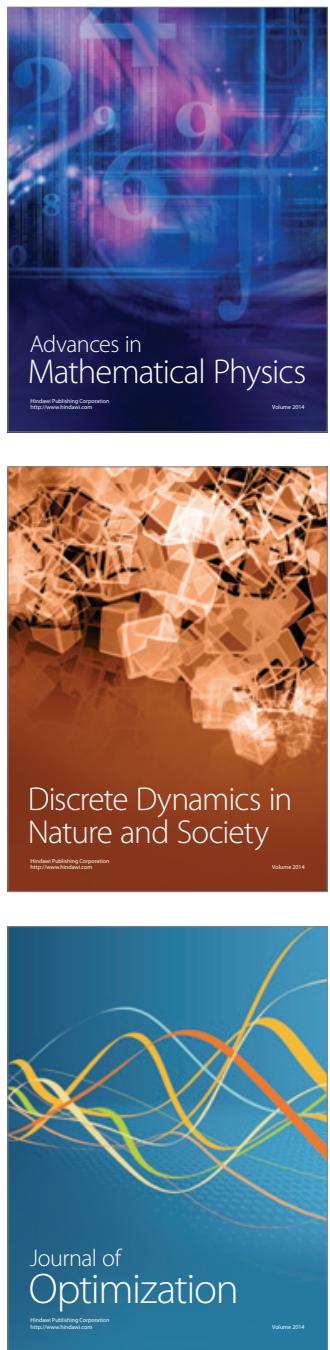